\title{
El perfil del voluntariado en la Biv@: análisis descriptivo desde 2006 hasta 2012
}

The profile of the Biv@volunteers: a descriptive analysis from 2006 to 2012

\author{
Ana Nieves MiLLÁn ReYes \\ Biblioteca Digital del Voluntariado y las Asociaciones (Biv@). Liga Giennense para la Educación y la Cultura Popular. \\ Avda., de Madrid, 46. Jaén (España), biva1@biva.es
}

\begin{abstract}
Resumen
La Biv@ nace del compromiso de la sociedad andaluza a través de la Agencia Andaluza del Voluntariado y asociaciones como la Liga Giennense vinculadas al tercer sector como una fuente de información especializada que facilita el acceso al conocimiento generado por el tercer sector, las asociaciones y el voluntariado. Tras seis años de andadura la Biv@ se ha consolidado como un recurso informativo, formativo y participativo. El número de voluntarios ha ido creciendo, pertenecen a ambos sexos por igual, predominan los que se encuentran entre los 20 y 30 años y los profesionales en activo, seguidos de los estudiantes.
\end{abstract}

Palabras clave: Bibliotecas digitales. Tercer sector. Voluntariado. Perfil de colaboradores.

\section{1. ¿Qué es la Biv@?}

La "Biblioteca Digital del Voluntariado y las Asociaciones" (BIV@), desde sus inicios hasta la actualidad se ha constituido en un importante recurso en el ámbito de los servicios no lucrativos y los servicios de información especializados, como instrumento de clasificación y almacenamiento, y como recurso útil para las personas e instituciones del voluntariado.

\section{2. ¿Qué ofrece?}

La Biv@ (Biblioteca Digital del Voluntariado y las Asociaciones) tal y como circunscribe su ámbito proporciona acceso a información organizada, accesible, relevante y permanente a todas las personas y entidades que se dedican a la promoción, al conocimiento o a la intervención voluntaria y asociativa. Tiene un marcado carácter de recurso público, gratuito, útil y de calidad para las personas que ejercen la acción voluntaria, la investigación, el estudio, la actividad técnica y profesional, las funciones directivas y la planificación en el amplio mundo del tercer sector.

La Biv@ ofrece diferentes servicios (catálogo, descargas de documentos, ofrece un directorio de asociaciones, recursos por y para el volunta-

\begin{abstract}
The Digital Library for Volunteers and Associations (Biv@) is sponsored by the Volunteers' Andalusian Agency and Third Sector associations, such as the Liga Giennense. It is a specialized information source that can facilitate access to pertinent knowledge from and by the third sector, associations and volunteers. After six years the Biv@ has established itself as an informative, educational and participatory resource. Volunteers are growing, men and women in a similar proportion, and mainly beteween 20 and 30 years old and professionally active or studying.
\end{abstract}

Keywords: Digital libraries. Third sector. Volunteering. Collaborators' profile.

riado y otros recursos relacionados, así como enlaces de interés, y un foro como herramienta de debate).

Así mismo, proporciona un espacio de participación ciudadana a través del perfil de voluntario o de usuario, previo registro como uno u otro; a los que permite la participación a través del servicio de aportaciones y sugerencias que pueden ser de diversa índole, documentos que consideren de interés para las asociaciones, el voluntariado o el tercer sector, noticias, actividades o eventos, sitios web, enlaces, participando del servicio de aportaciones y sugerencias. La Biv@ se constituye en un espacio de voluntariado-aprendizaje online, que aporta al voluntario las cualidades que ofrece la red, flexible y adaptado a las necesidades personales, profesionales, se puede realizar desde casa, o desde cualquier lugar desde el que se disponga de un ordenador y conexión (asociación, biblioteca, sala de ordenadores de la facultad, etc.).

El registro de una persona con un perfil de Voluntario de la Biv@ le permitirá el acceso previa identificación mediante usuario y contraseña:

- Acceder al servicio de descargas de documentos con un máximo de 20 descargas diarias. 
- Realizar aportaciones al fondo documental con un máximo de 20 aportaciones diarias.

- Participar en el desarrollo y mantenimiento de la Biv@ en las diferentes áreas propuestas (mantenimiento del foro, aportaciones al fondo documental, etc.,)

- Recibir la formación necesaria para orientarle en relación a que es ser y no ser voluntario, así como proporcionarle en aquellos casos que sea necesario unos conocimientos de partida para ejercer su voluntariado.

- Recibir la certificación y acreditación de los servicios prestados y de la participación en las actividades de voluntariado desarrolladas.

La colaboración como voluntario en la BIV@ está regulada por la Ley 7/2001 Andaluza del Voluntariado, en la que se especifican los derechos y deberes de la persona voluntaria, entre los que destacan para este propósito los referidos a rechazar cualquier contraprestación material como remuneración de sus actividades voluntarias, así como seguir las instrucciones técnicas en el desarrollo de las actividades que se les señalen por los responsables de la entidad de voluntariado.

La Biv@ a su vez constituye un espacio de aprendizaje y conocimiento compartido que contribuye a formar una base de conocimiento sobre el asociacionismo, el voluntariado por y el tercer sector. Posibilita la realización un aprendizaje-servicio, mediante que los voluntarios y usuarios contribuyen al proyecto y adquieren habilidades relativas a las áreas de voluntariado en las que pueden colaborar (catalogación, mantenimiento del foro, etc.) Aprendizajeservicio destinado a todas las edades y que puede personalizarse en función del voluntario. Cuyos beneficios se traducen en la mejora de las capacidades, mejora del sentido de la responsabilidad cívica y social, al contribuir tanto al proyecto como indirectamente a la comunidad a la que se orienta. Proporciona una aproximación diferente a las habilidades y competencias en un espacio no formal y que permite al estudiante, profesional, etc., en su faceta de voluntario acercarse a una realidad, a una visión práctica de sus conocimientos.

\section{3. ¿Quiénes pueden participar y como se puede participar?}

La Biv@ es un recurso abierto a todos los ciudadanos vinculados o no al movimiento asociativo, que deseen participar de forma activa en el mantenimiento, desarrollo del proyecto a través de las diversas formas de participación (voluntario, usuario) y de las diferentes espacios de participación propuestos. Se pueden beneficiar de una participación directa todos aquellos ciudadanos vinculados a asociaciones y organizaciones sociales que trabajan por y para el voluntariado: instituciones académicas, profesionales cualificados en áreas relacionadas con el asociacionismo, fundaciones, obras sociales, investigadores, estudiantes que deseen realizar un aprendizaje-servicio acreditado, así como los voluntarios que lo deseen.

Mediante el perfil de voluntario - que cuando lo desee se puede dar de baja- se puede participar en la dinamización, mantenimiento y actualización del Foro del Voluntariado; y en la actualización y aportación de noticias, eventos, actividades sobre el voluntariado, asociacionismo, tercer sector. Del mismo modo, se pueden realizar aportación de enlaces, sitios webs, direcciones, organizaciones de interés para el voluntariado, el asociacionismo y el tercer sector; incluir asociaciones $\mathrm{u}$ organizaciones de voluntariado; incrementar con la aportación de documentos el fondo documental; participar en la catalogación de documentos (en este caso se requiere que la persona voluntaria sea estudiante o tenga conocimientos sobre biblioteconomía, catalogación, clasificación); así como realizar sugerencias de mejora.

El perfil de usuario permite participar en el proyecto mediante la aportación de documentos, la inclusión de enlaces, actividades, eventos, asociaciones y realizar sugerencias entorno al proyecto. La Biv@ al ser un recurso online permite la participación ciudadana de aquellas personas que de forma voluntaria, o bien, como usuarios, de cualquier parte del mundo que lo deseen quieran participar de este proyecto.

\section{Breve panorámica de la Biv@}

Desde sus comienzos en el año 2006, la Biv@ - presentada en las XVI Jornadas Bibliotecarias de Andalucía (Rosa Díaz et al., 2007) — ha ido creciendo e incrementando progresivamente su fondo documental, que en la actualidad cuenta con unos 2281 documentos repartidos entre las diferentes materias que conforman la clasificación propia empleada en el catálogo y relativos a las asociaciones, el voluntariado y el tercer sector.

Esta evolución también ha favorecido el incremento de su número de usuarios que asciende a 988 y el mantenimiento del número de voluntarios. 
Esta evolución también se ha traducido en un incremento del tráfico de visitas total de 24.663 hasta el momento, procedentes de todo el mundo tal y como puede verse en el mapa de calor (figura 1) que ofrece el contador, pero primando la zona europea.

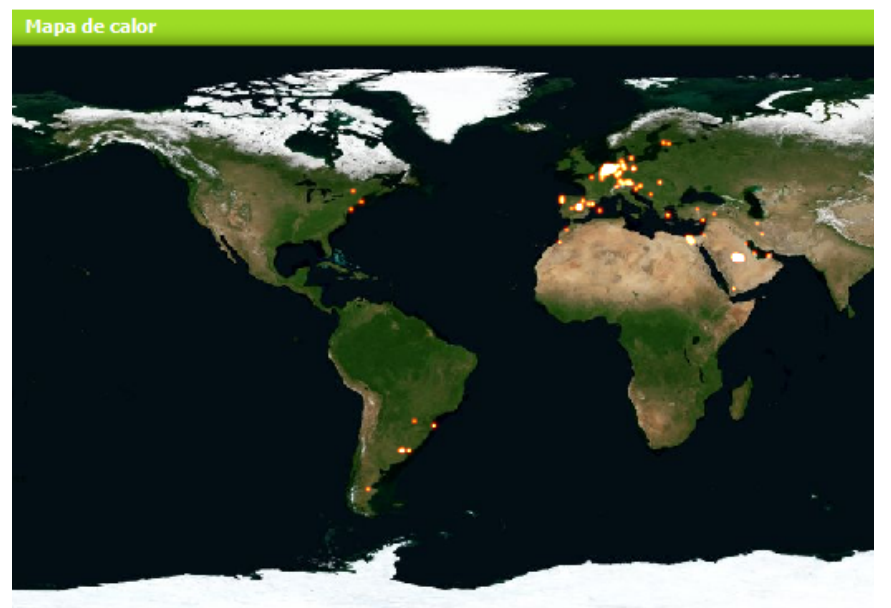

Figura. 1. Mapa de calor del contador de visitas.

En la gráfica 1, pueden verse los principales países visitantes.

$$
\begin{aligned}
& \text { 1. Países Bajos } \square \text { 2. Tailandia } \square \text { 3. Alemania } \square \text { 4. Arabia Saud } \\
& \text { 5. España } \square \text { 6. Marruecos } \square \text { 7. Egipto } \\
& \text { 9. Vietnam } \square \text { 10. Bélgica } \square \text { 10. El resto }
\end{aligned}
$$

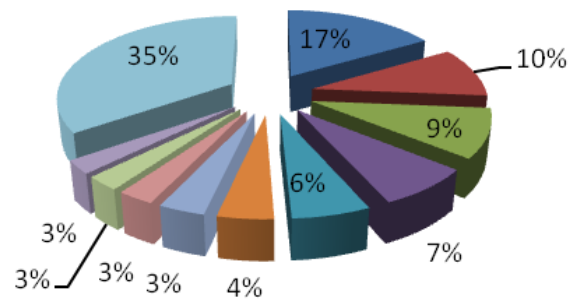

Gráfica 1. Principales países

La Biv@ ha logrado hacerse un hueco visible en la red, no obstante, ha de afrontar retos derivados de la evolución de ésta como incorporar su presencia en las redes sociales (facebook, twitter, etc.,) para incrementar el dinamismo y la comunicación entre ésta y sus usuarios, ofrecer nuevas oportunidades y espacios de participación; así como continuar con el estímulo hacia la incorporación de nuevos voluntarios, el mantenimiento y dinamización de los actuales, junto con la difusión del proyecto de participación ciudadana.

\section{El perfil del voluntariado en la Biv@s}

En la Biv@ las personas que se inscriben o desean dar de alta como voluntarios han de rellenar una ficha del voluntario con una serie de datos. A partir de los datos recogidos, se realiza la descripción del perfil del voluntariado.

El voluntariado dada las implicaciones, el compromiso que éste conlleva, y la situación de crisis que se viene arrastrando no ha evolucionado del mismo modo, en total unos 52. Este dato para los escepticos puede ser interpretado como una baja participación, pero para otros es un reflejo de una proporción de la participación voluntaria que se produce. Algunos autores indican que conocer la participación del voluntariado en España no es algo fácil pues es heterogéneo, complejo, cambiante (Zurdo Alaguero, 2011). Hemos de tener en cuenta que el voluntariado no es algo obligado, ni exigible; es una expresión de compromiso (Castellano Garrido, 2011). Consolidado normativamente (Benlloch, 2011), lo que permite obtener unas garantías, aunque no evitar perversiones. Debe ser entendido desde diversas corrientes o dimensiones conceptuales (Castellano Garridos 2011), lo que tal vez dificulte su entendimiento por la ciudadanía. Lo anterior unido a la situación de crisis que arrastramos ha provocado la ausencia de inscripciones de voluntarios en los años 2009 y 2010. En 2011 se han incrementado levemente, tal vez por la influencia de ser el Año Europeo del Voluntariado. No obstante, la incorporación de voluntarios ha ido creciendo (gráfica 2) repartidos en las diferentes áreas de colaboración, aportación de documentos, enlaces, noticias, mantenimiento del foro, etc.

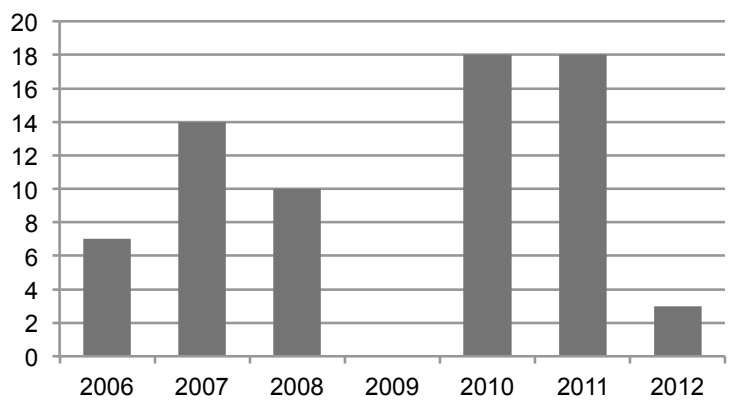

Grafica 2. Evolución de altas de voluntarios.

El análisis del perfil del voluntariado los analizaremos en función de las variables procedencia, profesión o actividad del voluntario, género, y comunidad autónoma de procedencia en el caso de los voluntarios cuya nacionalidad es la española. 
La procedencia de los voluntarios puede verse en la gráfica 3 , en la que el mayor porcentaje proviene de la España $88 \%$ de los voluntarios dados de alta, seguido Argentina 4\%, Venezuela, Portugal, Cuba o Estados Unidos con un $2 \%$.

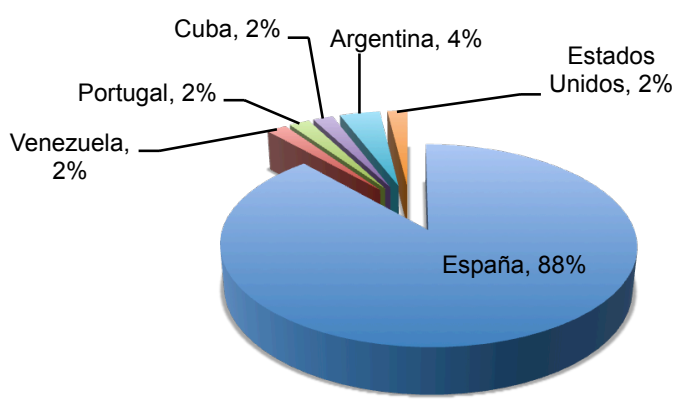

Gráfica 3. Procedencia

En relación al género el perfil de los voluntarios inscritos en situación de alta de la biva alcanzana una situacion paritaria. El porcentaje de hombres y mujeres es el mismo $50 \%$.
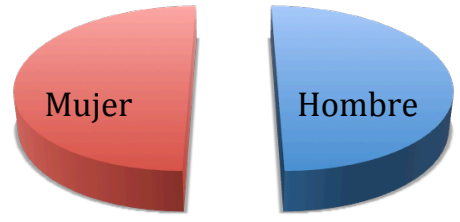

\section{Gráfico 4. Género}

La edad de inicio del periodo voluntario media es de 32,2. La distribución en porcentajes de las edades de inicio del periodo voluntario puede verse en la gráfica en la que se observa que la etapa más activa de inicio del período de voluntariado se observa entre jóvenes de 20 y 30 años dismunuyendo en intensisdad con el aumento de la edad.

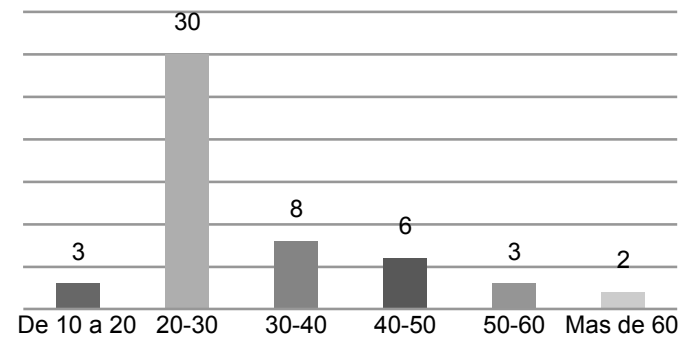

Gráfico 5. Edad de alta
Las principales áreas seleccionadas por las personas inscritas como voluntarios son las siguientes de las que ofrece la biv@ para colaborar distribuidas por año se observa que la catalogación los años 2006, 2007, 2011 junto con el mantenimiento del foro con mayor actividad en los años 2008, 2011. Para el área de programación la mayor actividad se ha dado en año 2011.

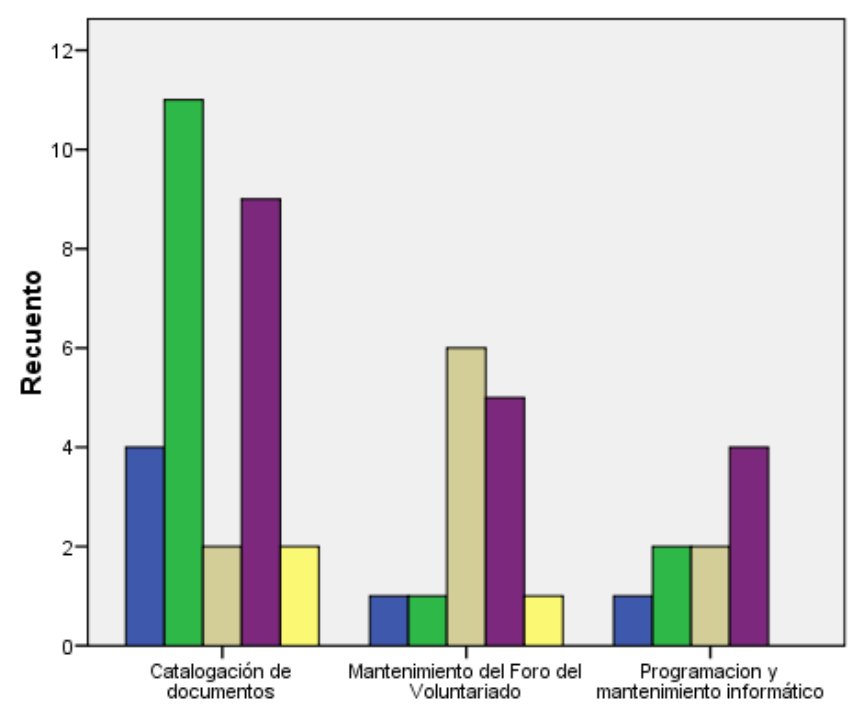

Gráfico 6. Área de participación

Un aspecto a tener en consideración es que no todos las personas inscritas tienen conocimientos previos relativos al área de voluntariado en la que participan. Resulta llamativo que la mayor parte de los voluntarios que solicitan el área de catalogación no provienen o tienen cualificación profesional específica en el área.

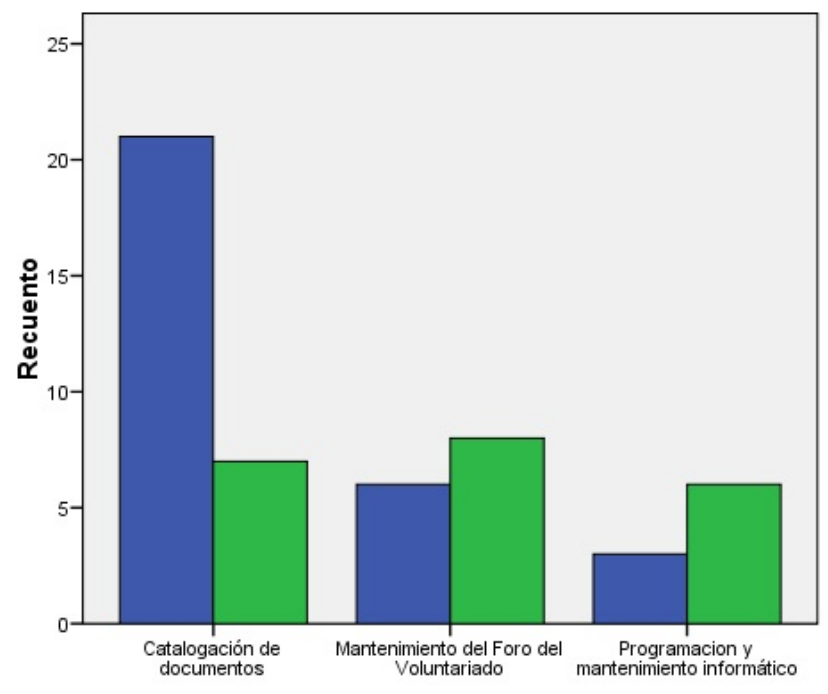

Gráfica 7. Conocimientos previos por área 
En cuanto a la duración del periodo de voluntariado por las personas inscritas el más frecuente es un voluntariado por meses, seguido del anual. En menor medida las personas inscritas como voluntarias manifiestan un periodo indefinido de voluntariado, es decir, que no tiene término señalado. El más escaso es el período por días. Tendencia que se observa en las tres áreas de voluntariado.

El área profesional de los voluntarios nos indica una mayor procedencia de profesionales en activo, seguido de profesionales con una cualificación superior, estudiantes en activo, y personas con una enseñanza media.

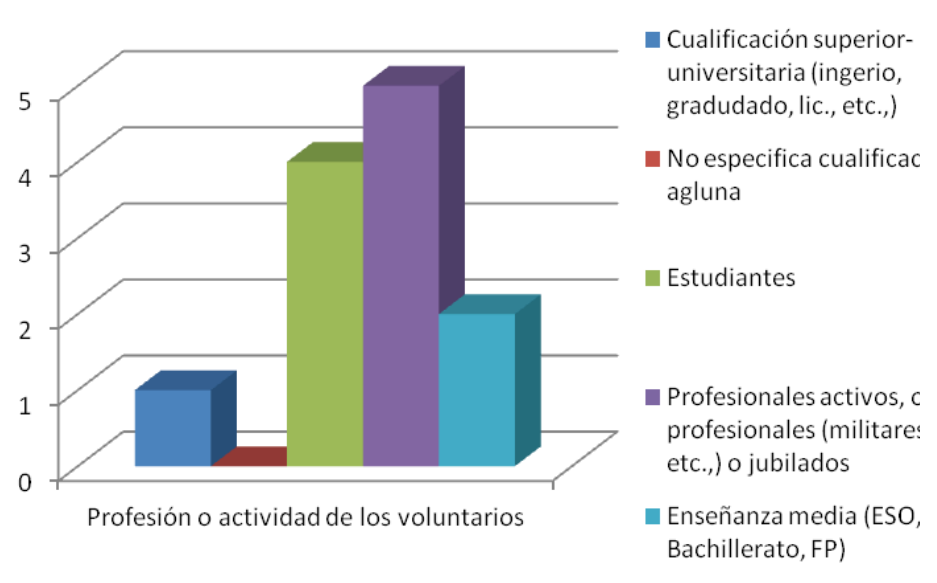

Gráfica 9. Cualificación de los voluntarios

\section{Retos y Conclusiones}

La Biv@ constituye un espacio abierto permanente en línea para que el ciudadano que lo desee pueda ejercer su compromiso social mediante la labor del voluntariado. Con la materialización de este compromiso social se contribuye al crecimiento del tercer sector, se genera un espacio acceso abierto de conocimiento compartido por y para el asociacionismo, el voluntariado y el tercer sector.

La Biblioteca Digital del Voluntariado y las Asociaciones es una plataforma en la que se desarrolla a través del aprendizaje-servicio una alfabetización informacional personalizada de acuerdo al tipo de voluntario o de voluntariado. Es un recurso con una vocación orientada a facilitar el aprendizaje permanente 0 a lo largo de toda la vida al proporcionar contenidos especializados por y para el voluntariado, el asociacionismo y el tercer sector, y a partir de la creación de redes informales para compartir experiencias y conocimientos.

Constituye una herramienta para la cooperación y el desarrollo.

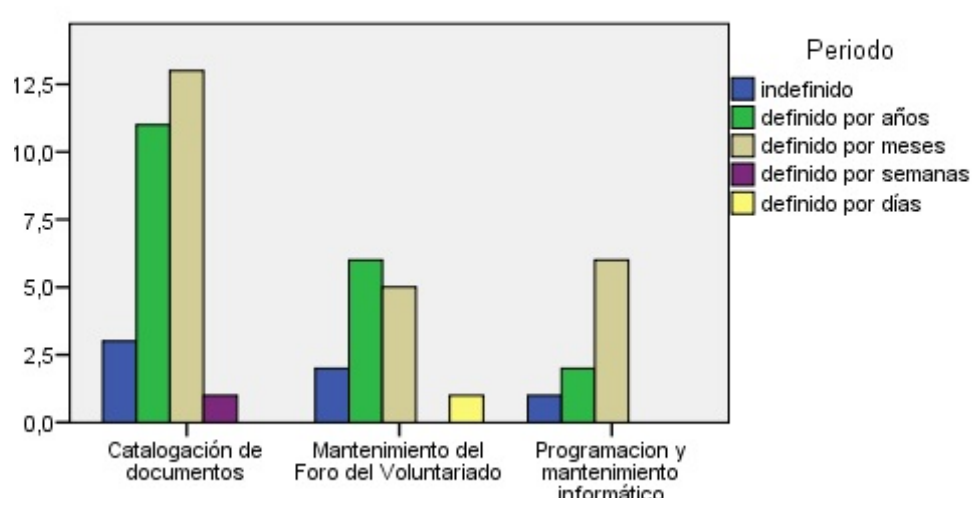

Gráfica 8. Actividades

La Biv@ constituye una iniciativa enmarcada en el compromiso social, por y para el voluntariado, el asociacionismo y el tercer sector, fruto de la responsabilidad social tanto de las organizaciones sin ánimo de lucro como de los profesionales de la información de crear contenidos de calidad, accesibles para la sociedad, dada "la importancia moral de ofrecer un entorno rico en información" (Candas, 2010, p. 58).

La consolidación y el mantenimiento de los voluntarios constituyen dos de los principales retos, máxime en tiempos de crisis; y especialmente en un tiempo de crisis de valores, en el que el voluntariado se puede ver menospreciado, pervertido por los políticos, o malentendido por la sociedad.

\section{Referencias}

Arias Careaga, S. (2011). Voluntariado Universitario: estado de la cuestión. Revista Española del Tercer Sector, N018. http://www.fundacionluisvives.org/rets (2012-02-8)

Arnaz Villalta, E. (2011). Voluntariado y participación. Revista Española del Tercer Sector, №18. http://www.fundacionluisvives.org/rets (2-02-2012)

Benlloch Sanz, P. (2011). Una nueva oportunidad para repensar el voluntariado: ¿Hacia una nueva configuración legal de la acción voluntaria organizada?. // Revista Española del Tercer Sector. 18. http://www.fundacionluisvives.org/rets (18-01-2012)

Candás-Romero, J. (2010). Ética profesional en Biblioteconomía, Universidad Carlos III de Madrid [Tesis doctoral]. http://www.uc3m.es (09-06-2011).

Castellano Garrido, I.; et al. (2011). Voluntariado: Tendencias y Retos (en España y hoy). // Revista Española del Tercer Sector, $\mathrm{N}^{\circ} 18$. [en línea]. Disponible en.: http://www.fundacionluisvives.org/rets (12-09-2011)

Flores Martos, R. (2011). Características del Voluntariado de Acción Social. Revista Española del Tercer Sector, №18. http://www.fundacionluisvives.org/rets (10-102011)

Rosa Díaz, A.; et al. (2007). La Biblioteca Digital del Voluntariado y las Asociaciones: una herramienta para la integración y el crecimiento social. // XIV Jornadas Bibliotecarias de Andalucía. Antequera (Málaga) 15 al 17 de marzo de 2007. Más que palabras: las bibliotecas motor 
de transformación social. http://www.aab.es/pdfs/jba14/ Comunicaciones/Millan.pdf (14-06-2011)

Zurdo Alaguero, A. (2011). Voluntariado y procesos democráticos en las sociedades contemporáneas. Revista
Española del Tercer Sector. 18.http://www.fundacion luisvives.org/rets (20-01-2012).

Enviado: 2012-04-15. Aceptado: 2012-07-02. 